\title{
Genomic relationships between selected phage types of Salmonella enterica subsp. enterica serotype typhimurium defined by ribotyping, IS200 typing and PFGE
}

\footnotetext{
1 Department of Veterinary Microbiology, The Royal Veterinary and Agricultural University, Bülowsvej 13, DK-1870 Frederiksberg C, Denmark

2 Laboratory of Enteric Pathogens, Central Public Health Laboratory, 61 Colindale Avenue, London NW9 5HT, UK
}

\author{
John E. Olsen, ${ }^{1}$ Marianne N. Skov, ${ }^{1}$ Øystein Angen, ${ }^{1}$ E. John Threlfall ${ }^{2}$ \\ and M. Bisgaard'
}

Author for correspondence: John E. Olsen. Tel: +45352 82784. Fax: +4535282757. e-mail: John.E.Olsen@vetmi.kvl.dk
The genomic relationship between isolates representing 17 definitive phage types (DTs) of Salmonella enterica subsp. enterica serotype typhimurium (S. typhimurium) were analysed using three different typing methods: IS200 typing using the restriction enzymes EcoRI and Pvull, ribotyping using Smal and EcoRI, and PFGE using Xbal. These methods were used to study four DTs in greater detail; in all 18 (DT 49), 10 (DT 110), five (DT 120) and seven (DT 135) isolates were studied. The combined data generated two large clusters, which could be divided into five groups. Within the first cluster, a close similarity was indicated between isolates of the following phage types: group A - DTs 44, 49, 135 and 204c, with DT 9 distantly related; group B - DTs 95 and 99; and group C - DTs 104a, 110 and 120. The other large cluster contained group D - DTs 10, 20 and 146, with DT 12 distantly related, and group E-DTs 69, 103 and 153. The same grouping was observed by principal component analysis, but a minimum spanning tree linked DT 12 to group E and not group D in this analysis. Among the typing methods used, IS200 gave the best representation of the overall similarity between the S. typhimurium isolates. Five different IS200 profiles were obtained among isolates belonging to DT 49. Only one profile was observed within each of the phage types DT 110, 120 and 135. All isolates within each of these four phage types were of one ribotype. Isolates of DT 49 showed four PFGE patterns, while one pattern was present within isolates of the three other phage types. Members of these four phage types were found to be clonally related as they formed tight subclusters separated from isolates of other phage types.

Keywords: clonality, IS200, ribotype, pulsed-field gel electrophoresis, Salmonella typhimurium

\section{INTRODUCTION}

Salmonella enterica subsp. enterica serotype typhimurium (denoted S. typhimurium throughout this paper) is a common cause of salmonellosis among humans and animals in many countries. Phage typing by the method of Callow (1959) is normally used for identification of this serotype, and more than 200

Abbreviations: DT, definitive phage type; MST, minimum spanning tree; UPGMA, unweighted-pair group method with arithmetic averages. definitive phage types (DTs) have been recognized (Anderson et al., 1977). However, only one or a few phage types tend to dominate within a geographical region for a period of time, presumably due to spread of a successful clone or due to the infrastructure of animal production systems. For instance, S. typhimurium DT $204 \mathrm{c}$ remained an important cause of salmonellosis among both cattle and humans in the UK throughout the 1980s (Threlfall et al., 1990a), but has subsequently become less common in both bovines and humans. Likewise, $70 \%$ of isolates obtained from clinically diseased pigs in Denmark in the second half of 1992 
Table 1. Isolates of $S$. typhimurium used as representative of a DT

\begin{tabular}{|rll|}
\hline DT & Isolate & \multicolumn{1}{c|}{ Source* } \\
\hline 9 & P79340 & LEP, human, 1988 \\
10 & P79352 & LEP, human, 1988 \\
12 & P98968 & LEP, human, 1989 \\
20 & P79182 & LEP, human, 1989 \\
44 & P91453 & LEP, human, 1989 \\
49 & P124218 & LEP, bovine \\
69 & P100185 & LEP, human, 1985 \\
95 & $23 M 8909$ & LEP, human, 1984 \\
99 & P98192 & LEP, human, 1989 \\
103 & P93660 & LEP, human, 1989 \\
$104 \mathrm{a}$ & P98849 & LEP, human, 1989 \\
110 & $7378911-2$ & NVL, poultry \\
120 & NVL8653 & NVL, poultry \\
135 & JEO3673 & LEP, unknown \\
146 & P98020 & LEP, human, 1989 \\
153 & P96845 & LEP, human, 1989 \\
$204 \mathrm{c}$ & $23 M 5519$ & LEP, bovine, 1983 \\
\hline
\end{tabular}

* LEP, Laboratory of Enteric Pathogens, Central Public Health Laboratory, UK; NVL, National Veterinary Laboratory, Denmark.

belonged to a single phage type (DT 12), while $65 \%$ of poultry isolates belonged to DTs 110 or 135 (Baggesen $\&$ Wegener, 1994).
New phage types of $S$. typhimurium have been shown to evolve from recognized types through defined genetic changes. Thus, the cattle-associated multidrug-resistant DT 204c developed from a drug-sensitive strain of DT 49 as a result of the acquisition of plasmids, transposons and temperate phages (Threlfall et al., 1983). In most cases, however, the genomic relationship between different phage types is unknown. The relationship between phage types of $S$. typhimurium has recently been investigated using IS200 profiling and ribotyping, and a phylogenetic tree based on the copy number of IS200 and identity of ribotype patterns was produced by Stanley et al. (1993). In the present study, this approach has been investigated further by quantitative comparison of IS200, ribotype, and PFGE patterns of isolates belonging to 17 different phage types of $S$. typhimurium. As only limited information is available on genetic diversity within a single phage type, this was investigated by analysing a number of isolates belonging to the commonly isolated phage types DT 49,110,120 and 135 using molecular methods.

\section{METHODS}

Bacterial isolates and media. Strains characterized as representatives of individual phage types in the present study are listed in Table 1. Additional strains analysed to investigate the genetic diversity within DTs 49,110,120 and 135 are listed in Table 2. They were propagated overnight in LB medium (Maniatis et al., 1982) at $37^{\circ} \mathrm{C}$, and stored at $-80^{\circ} \mathrm{C}$ in $\mathrm{LB}$ supplemented with $15 \%(\mathrm{v} / \mathrm{v})$ sterile glycerol between analy-

\section{Table 2. Additional strains of DTs $49,110,120$ and 135 analysed}

Strains of DT 49 are representatives of 18 out of 20 different plasmid profiles identified among strains of this phage type. The strains were isolated in the UK in 1984-1989 (Threlfall et al., 1990b). Strains of DTs 110, 120 and 135 were isolated in Denmark in 1993 and 1994.

\begin{tabular}{|lll|lll|}
\hline DT & Strain & Source & DT & Strain & Source \\
\hline 49 & P137477 & LEP, avian & 110 & 9412045 & NVL, porcine \\
49 & P129881 & LEP, human & 110 & 9422387 & NVL, porcine \\
49 & P139489 & LEP, human & 110 & 9421647 & NVL, porcine \\
49 & P64913 & LEP, human & 110 & 9413689 & NVL, bovine \\
49 & P122475 & LEP, human & 110 & 9422550 & NVL, porcine \\
49 & P93752 & LEP, human & 110 & 9422676 & NVL, porcine \\
49 & P97255 & LEP, human & 110 & 9414252 & NVL, bovine \\
49 & P124420 & LEP, human & 110 & 9422887 & NVL, porcine \\
49 & $20 M 3706$ & LEP, bovine & 110 & 9416044 & NVL, bovine \\
49 & $20 M 4748$ & LEP, bovine & 120 & SVS1107 & NVL, meat \\
49 & P56183 & LEP, human & 120 & SVS10658 & NVL \\
49 & P50703 & LEP, human & 120 & SVS1028 & NVL, bovine \\
49 & P13719 & LEP, human & 120 & SVS5188 & NVL, poultry \\
49 & P148106 & LEP, human & 135 & SVS1101 & NVL, meat \\
49 & P124483 & LEP, human & 135 & SVS11081 & NVL, human \\
49 & P132189 & LEP, human & 135 & SVS11235 & NVL, poultry \\
49 & P130646 & LEP, human & 135 & SVS11237 & NVL, poultry \\
& & & 135 & SVS485 & NVL, bovine \\
& & 135 & SVS6443 & NVL, porcine \\
\hline
\end{tabular}


(a)

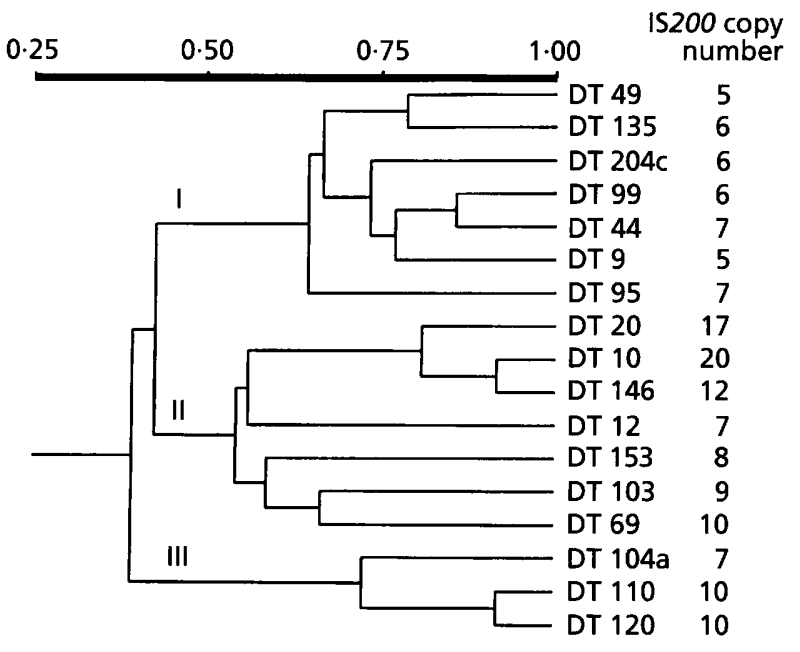

(b)

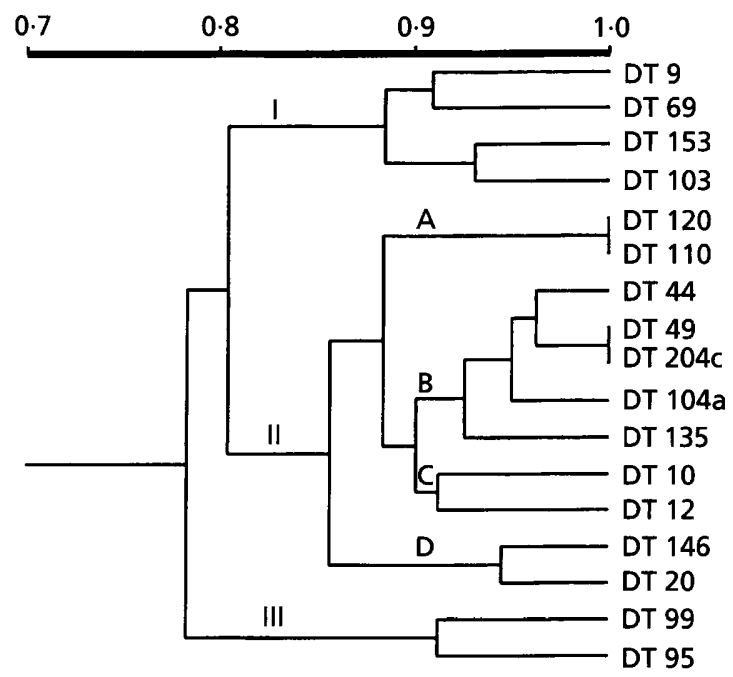

(c)

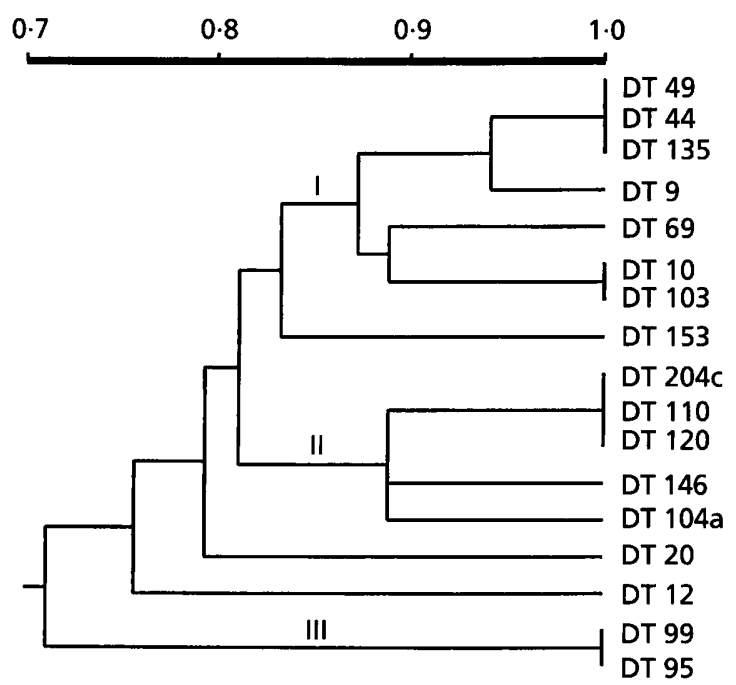

Fig. 1. Similarities between (a) IS200 (b) ribotype and (c) PFGE patterns of S. typhimurium. (a) Comparison was based on Pvulland ECORI-digested DNA probed with IS200, and results from ses. Strains of DTs 110,120 and 135 were kindly provided by Dr Dorte Lau Baggesen, National Veterinary Laboratory, Denmark. All other strains were from the strain collection at the Laboratory for Enteric Pathogens, Central Public Health Laboratory, UK.

IS200 typing. Total DNA was isolated from $1.5 \mathrm{ml}$ of an overnight broth culture by a neutral SDS method as described by Christensen et al. (1993). DNA was digested in separate reactions with restriction enzymes PvuII and EcoRI (Boehringer Mannheim or Amersham) as recommended by the supplier. DNA fragments were subsequently separated by agarose gel electrophoresis overnight at $1 \mathrm{~V} \mathrm{~cm}^{-1}$ in $0.8 \%$ (w/v) agarose gels (SeaKem GTG) prepared with TAE buffer (40 mM Tris/acetate, $1 \mathrm{mM}$ EDTA). Digoxigenin-labelled phage lambda DNA digested with restriction enzyme HindIII (Boehringer Mannheim) served as the molecular mass marker. Gels were stained in an aqueous solution of $2 \mu \mathrm{g}$ ethidium bromide $\mathrm{ml}^{-1}$ and photographed under UV light $(254 \mathrm{~nm})$. The DNA fragments were transferred to Hybond-N hybridization membranes (Amersham) by vacuum blotting as recommended by the supplier of the vacuum blotter (Pharmacia), and fixed to the membrane by baking at $80^{\circ} \mathrm{C}$ for $30 \mathrm{~min}$. A $300 \mathrm{bp}$ internal fragment of the insertion sequence IS200 (Gibert et al., 1990) was generated by PCR from a strain of Escherichia coli HB101 carrying the fragment cloned into plasmid pGEM3 as previously described (Olsen \& Skov, 1994). The PCR fragment was subjected to gel electrophoresis in $1.2 \%(\mathrm{w} / \mathrm{v})$ agarose, and the probe fragment was purified from the agarose with a Prep-a-Gene DNA-binding matrix (Bio-Rad). The fragment was labelled with digoxigenin-11-dUTP using the Random Primed labelling kit (Boehringer Mannheim). Filters were prepared for hybridization by incubation in hybridization solution [ $5 \times$ SSC (Maniatis et al., 1982), $1 \%$ blocking reagent (Boehringer Mannheim), $0.1 \% \mathrm{~N}$-lauryl sarcosine, $0.02 \%$ SDS] at $65^{\circ} \mathrm{C}$ for $2 \mathrm{~h}$, whereafter labelled probe was added and the hybridization carried out overnight at $65^{\circ} \mathrm{C}$. Posthybridization washes were done twice at room temperature and once for $30 \mathrm{~min}$ at $65^{\circ} \mathrm{C}$ in $5 \times \mathrm{SSC} / 0 \cdot 2 \%$ SDS. Blocking of filters and detection of hybridization signals were performed as recommended by Boehringer Mannheim. All strains were tested twice with both restriction enzymes, and no differences were observed between patterns. Restriction enzyme PvuII does not cut internally in IS200 (Stanley et al., 1991) and the hybridization patterns obtained with this enzyme were used to measure the copy number of the element in the chromosome.

Ribotyping. DNA isolation, restriction digestion, agarose gel electrophoresis, blotting, hybridization and detection of hybrids were essentially carried out as described for IS200 typing. Restriction enzymes SmaI and EcoRI (Boehringer Mannheim or Amersham) were used for this typing method. The digoxigenin-11-dUTP-labelled cDNA probe was synthesized from $16 \mathrm{~S}$ and $23 \mathrm{~S}$ ribosomal RNA from E. coli MRE600 using M-MLV transcriptase (Gibco BRL). The labelling was performed at $37^{\circ} \mathrm{C}$ for $2 \mathrm{~h}$ in a solution containing $50 \mathrm{mM}$ Tris/ $\mathrm{HCl} \mathrm{pH} 8 \cdot 3,75 \mathrm{mM} \mathrm{KCl}, 3 \mathrm{mM}$

the two analyses were pooled. (b) In separate analyses, Smal and EcoRI were used for ribotyping, and results from the two analyses were pooled. (c) Xbal was used for typing, and only bands larger than $100 \mathrm{~kb}$ were included in the analyses to eliminate the influence from plasmid bands. Similarity analysis was performed using the Dice coefficient and clustering was by UPGMA. 
Table 3. Variation obtained with different typing methods within DTs $49,110,120$ and 135 of S. typhimurium

\begin{tabular}{|cccc|}
\hline Method & $\mathrm{DT}^{*}$ & $\begin{array}{c}\text { Restriction } \\
\text { enzyme }\end{array}$ & $\begin{array}{c}\text { No. of types } \\
\text { (per phage type) }\end{array}$ \\
\hline IS200 typing & 49 & PvuII & 3 \\
& & EcoRI & 4 \\
& $110,120,135$ & PvuII/EcoRI & 4 \\
Ribotyping & $49,110,120,135$ & PvuII & 1 \\
& & EcoRI & 1 \\
PFGE typing & 49 & EcoRI & 1 \\
& $110,120,135$ & $X b a \mathrm{I}$ & 4 \\
& & $X b a \mathrm{I}$ & 1 \\
\hline
\end{tabular}

*Number of isolates analysed was: 18 (DT 49), 10 (DT 110), 5 (DT 120) and 7 (DT 135).

$\mathrm{MgCl}_{2}, 0.5 A_{260}$ unit $\mathrm{ml}^{-1} 16 \mathrm{~S}$ and 23S RNA (Boehringer Mannheim), $\quad 0 \cdot 13 \mu \mathrm{mol} \mathrm{m}^{-1}$ random primer $\left[\mathrm{p}(\mathrm{dN})_{6}\right]$ (Boehringer Mannheim), $25 \mathrm{nmol} \mathrm{ml}^{-1}$ each of dATP, dCTP and dGTP, $16 \mathrm{nmol} \mathrm{ml}^{-1}$ dTTP (Gibco BRL), $9 \mathrm{nmol}$ digoxigenin-11-dUTP $\mathrm{ml}^{-1}, 10 \mathrm{mM}$ DTT (Gibco BRL), 120 units RNase inhibitor $\mathrm{ml}^{-1}$ (Boehringer Mannheim), and 5000 units $\mathrm{M}-\mathrm{MLV} \mathrm{ml} \mathrm{l}^{-1}$. The labelled cDNA was precipitated with $96 \%$ ethanol, the pellet was washed twice with $70 \%$ ice-cold ethanol and resuspended in 1.25 ml TE 10:1 (10 mM Tris $/ \mathrm{HCl}$ $\mathrm{pH} 7 \cdot 6,1 \mathrm{mM}$ EDTA) and stored at $-20^{\circ} \mathrm{C}$. For each hybridization, $12.5 \mu$ l of this solution was used as probe. Prehybridization, hybridization and post-hybridization washes at high temperature were carried out at $56^{\circ} \mathrm{C}$. All strains were tested twice with each restriction enzyme. Weak bands differed between the gels, while strong bands were fully reproducible. Consequently, only strong bands were scored.

PFGE. DNA for PFGE was isolated by a procedure which enables the isolation of high-molecular-mass fragments of the chromosome (Cameron et al., 1994). Slices of agarose blocks containing the DNA were digested for $4 \mathrm{~h}$ with restriction enzyme $\mathrm{XbaI}$ (Amersham). DNA fragments were separated in $1 \%(\mathrm{w} / \mathrm{v})$ agarose gels in $0.5 \times \mathrm{TBE}$ buffer $(0.089 \mathrm{M}$ Tris/ borate, $0.089 \mathrm{M}$ boric acid, $2 \mu \mathrm{M}$ EDTA) in a contour-clamped homogeneous electric field system (Pharmacia) using running conditions described by Olsen et al. (1994). To eliminate the possible influence of large plasmids on the PFGE patterns, plasmids were isolated using the method of Olsen (1990) and digested with $\mathrm{Xba \textrm {I }}$. Large plasmids in all isolates were demonstrated to be cut by XbaI to fragments less than $100 \mathrm{~kb}$ in size, and only PFGE fragments larger than $100 \mathrm{~kb}$ were scored in the numerical analysis. All strains were run at least twice. Weak bands that could not be reproduced were interpreted as incomplete digestions and were ignored.

Numerical analysis. Gels and hybridization filters were scored manually. Each strain was given a score of 1 (present) or 0 (not present) for each possible band position. A numerical analysis of similarity in band patterns was performed using the software package NTSYs-pc (Rohlf, 1993). Four different datasets were included: IS200 patterns, ribotypes, PFGE profiles, and a dataset where data from all three techniques were pooled (IRP dataset). Similarities were analysed using the Dice coefficient and the unweighted-pair group method using average linkages (UPGMA) clustering (Sneath \& Sokal, 1973). Co-phenetic correlation coefficients, $r_{\mathrm{cs}}$, were calculated for the resulting dendrograms (Sneath \& Sokal, 1973). If the clustering program drew more than one graphic presentation of a matrix, the dendrogram with the highest $r_{\text {cs }}$ was chosen. The correlation between the four similarity matrices was computed using the MXcomp module in NTSYs. Further, a principal coordinate analysis (Gower, 1966) was performed on the Dice similarity matrix based upon the IRP dataset. A minimum spanning tree (MST) was superimposed upon the resulting ordination thus simultaneously displaying the similarities between the phage types as approximated by both the clustering and the ordination procedure.

\section{RESULTS}

Isolates of $S$. typhimurium were estimated to carry between five and 20 copies of IS200 (Fig. 1a). A clustering based on the similarity of IS200 patterns of isolates representing 17 different phage types of $S$. typhimurium is shown in Fig. 1(a) $\left(r_{\mathrm{cs}}=0.88\right)$. The analysis was based on two restriction enzymes and a total of 73 different fragment sizes. Three major clusters, which joined at approximately $40 \%$ similarity, were identified. Cluster I is a large group containing DTs 9, 44, 49, 99, 135 and $204 \mathrm{c}$, and more distantly related to this group, DT 95 ; cluster II comprises two groups containing DTs 10, 20, 146 and DTs 69,103 , respectively, and the more distant DTs, 12 and 153; and cluster III comprises DTs 110, 120 and 104a. As shown in Table 3, the 18 isolates of DT 49 had a total of four different IS200 patterns (two enzymes), indicating a degree of genetic variability within this phage type. Two isolates carried six copies of IS200 while the remaining strains had a copy number of five. Twelve isolates showed the same pattern. In a similarity analysis which included all four IS200 patterns, all members of DT 49 were found to form a tight subcluster within cluster I of Fig. 1(a) (data not shown). The strain selected to represent DT 49 in the comparison with 16 other DTs was of the common IS200 type. The different IS200 patterns of DT 49 are shown in Fig. 2(a). Isolates within each of the phage types DTs 110, 120 and 135 showed only one IS200 pattern. 
(a)

ECORI

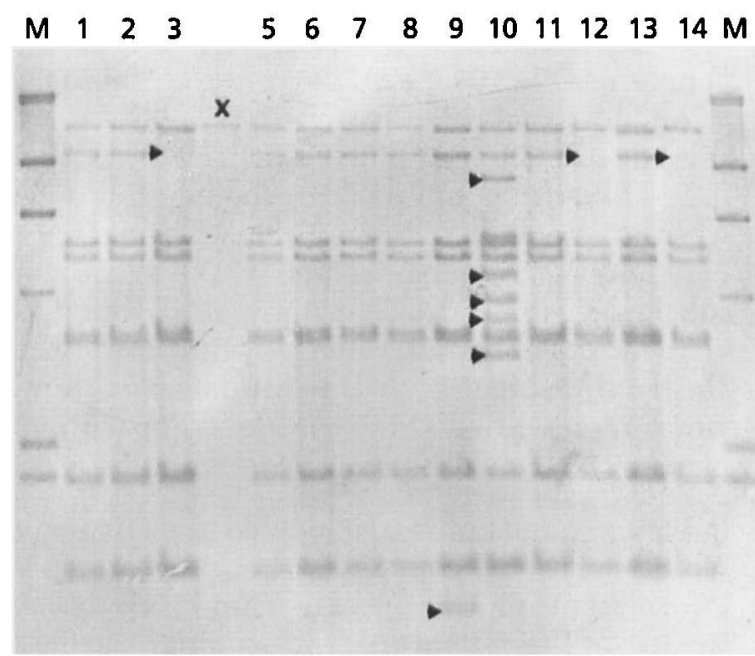

(b)

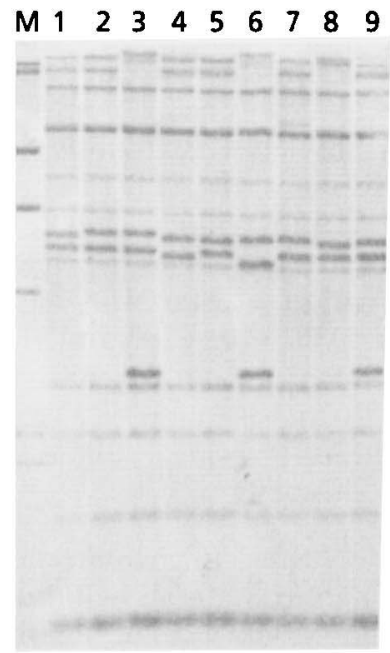

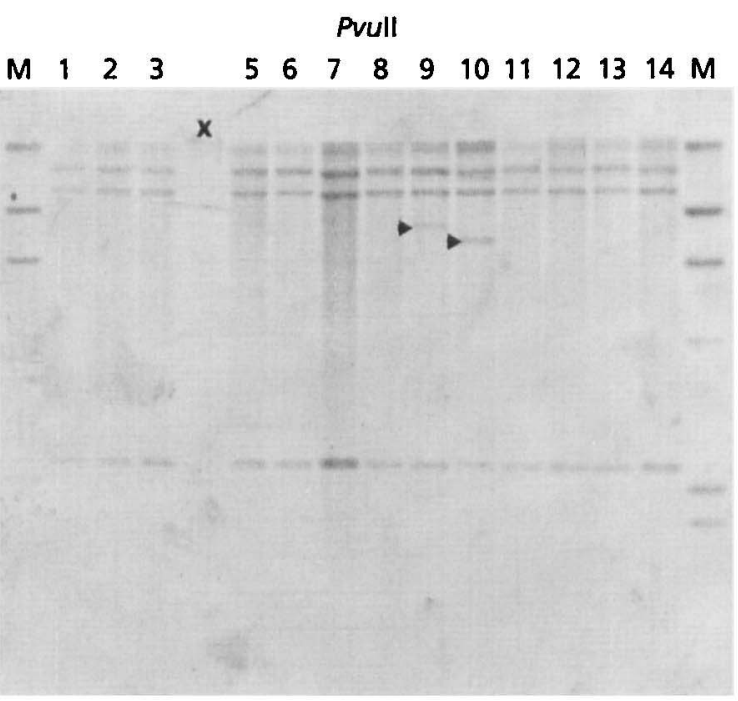

(c)

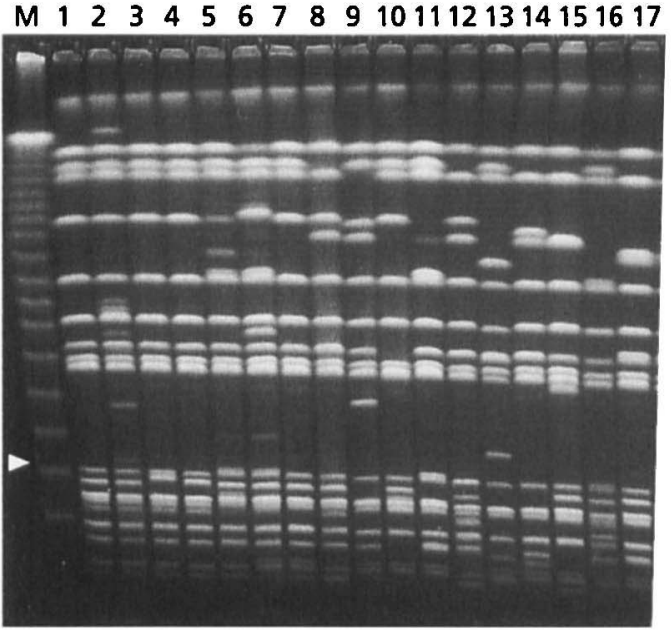

Fig. 2. (a) IS200 patterns of DT 49 of S. typhimurium after digestion with EcoRI (left) and Pvull (right). Lanes $X$ do not contain a strain of DT 49. Arrowheads indicate bands that differ from the general IS200 profiles of DT 49. Strains were in lanes: 1, P93752; 2, P77255; 3, P124420; 5, P122475; 6, 20M3706; 7, 22M4748; 8, P956183; 9, P50703; 10, P137913; 11. $\mathrm{P} 148106 ; 12, \mathrm{P} 124483 ; 13, \mathrm{P} 132189 ; 14, \mathrm{P} 130646$. (b) Smal ribotype patterns of selected DTs of $S$. typhimurium. Lanes: 1 , DT 49; 2, DT 20; 3, DT 9; 4, DT 10; 5, DT 204c; 6, DT 153; 7, DT 146; 8, DT 104a; 9, DT 103. In (a) and (b) $M$ indicates lambda/HindIII DNA molecular mass markers. (c) PFGE patterns of DT 49 and other selected DTs of S. typhimurium. Lane $\mathrm{M}$ contains the $50 \mathrm{~kb}$ molecular mass ladder. Only bands larger than $100 \mathrm{~kb}$ (arrowhead) were included in the similarity analysis. Lanes 1-8 show PFGE patterns of DT 49: 1, P124218; 2, P139489; 3, P124420; 4, P130646; 5, P956183; 6, P137913; 7, P124483; 8, P132189. Lanes 9-17 show different DTs: 9, DT 20; 10, DT 9; 11, DT 10; 12, DT 204c; 13, DT 153; 14, DT 146; 15, DT 104a; 16, DT 103; 17, DT 99. Weak bands in lane 2 are not reproducible and were ignored in the comparisons of PFGE types. The strains of DT 49 where the PFGE type differs from the type normally seen with this DT are shown in lanes 5,6 and 8 .

The analysis of ribotype similarities with two different enzymes was based on a total of 44 fragment sizes. Two dendrograms were drawn from the similarity matrix of which the dendrogram shown in Fig. 1(b) had the highest $r_{\text {cs }}$ value $(0 \cdot 86)$. One large and two small clusters were identified, joining at approximately $78 \%$ similarity. The small clusters [Fig. 1(b) cluster I and III] contained the following phage types: (I) DTs 9, 69, 103 and 153, and (III): DTs 95 and 99. The remaining isolates belonged to the large cluster (Fig. 1(b) cluster II) and were divided into four groups (Fig. 1(b) cluster II, groups A-D). Group A contained DTs 110 and 120 . Groups B and C formed a subcluster where all types had $90 \%$ or more ribotype similarity. Group B contained DTs 44, 49, 104a, 135 and 204c and group C contained DTs 10 and 12. Group D contained DTs 20 and 146. All 
(a)
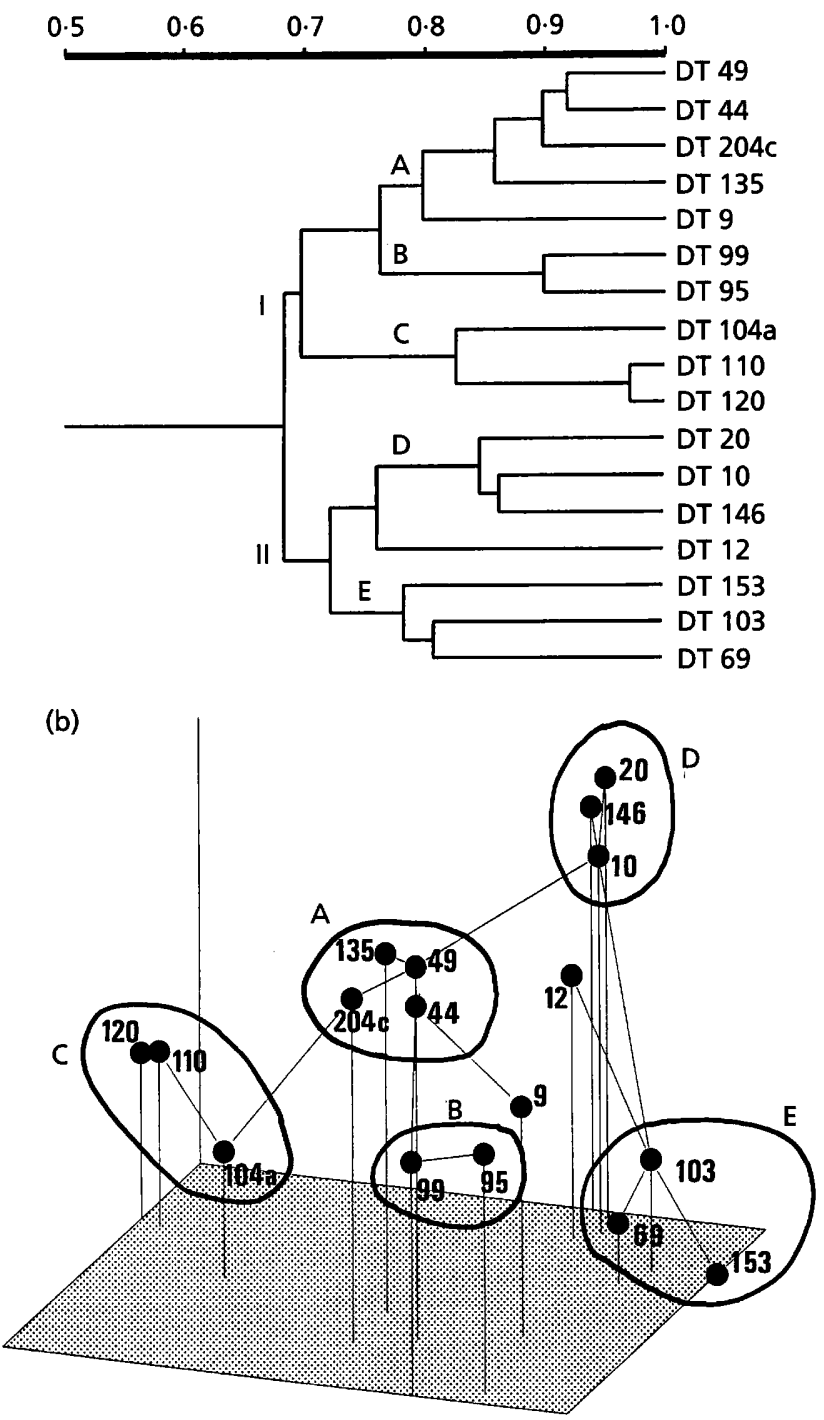

Fig. 3. (a) Overall similarities between restriction fragment patterns of $S$. typhimurium. Bands produced by IS200, ribo- and PFGE typing were included. Similarity analysis was performed using the Dice coefficient and clustering was by UPGMA. (b) Principal component analysis of the overall similarities between restriction fragment patterns of $S$. typhimurium produced by IS200 typing, ribotyping and PFGE typing. The figure shows the distances between the phage types in the space spanned by the principal axes as well as the nearest neighbours of the individual phage types as indicated by the MST. The groups are encircled and numbered according to the UPGMA analysis in (a).

isolates within DTs $49,110,120$, and 135 , respectively, were of one SmaI and EcoRI ribotype (Table 3). Some ribotype patterns are shown in Fig. 2(b).

The analysis of PFGE similarities was based on 20 different fragment sizes. The clustering (Fig. 1(c), $r_{\mathrm{cs}}=$ 0.83 ) resulted in the formation of two clusters (Fig. 1c, I and II), which joined at approximately $80 \%$ similarity.
Cluster I contained DTs 9, 44, 49 and 135 and DTs 10, 69 and 103. DT 153 belonged to this group but was only distantly connected to these phage types. Cluster II contained DTs 104a, 110, 120, 146 and 204c. DTs 12, 20, 95 and 99 remained unclustered. DTs 95 and 99 showed the same PFGE type (Fig. 1c, III). PFGE patterns were found to differ within DT 49 where four different types were observed (Fig. $2 \mathrm{c}$ and Table 3). Unlike the analysis based on IS200 typing, isolates of DT 49 did not form a subcluster in a dendrogram in which all four PFGE types were included, but intermingled with clusters I and II or clustered together with DT 12 (data not shown). The strain representing DT 49 in the comparison to other phage types was of the most common PFGE type.

It was assumed that the pooled data from all three typing methods contained the most comprehensive information on the genetic similarity between the isolates of S. typhimurium. In a comparison based on all three methods, two large clusters were identified (Fig. $3 \mathrm{a}, \mathrm{I}$ and II $; r_{\mathrm{cs}}=0.79$ ), which joined at approximately $67 \%$ similarity. Cluster I contained two subclusters $(A B$ and C) which joined at approximately $70 \%$ similarity; the largest subcluster was further divided into two groups (Fig. 3a, A and B) which joined at approximately $76 \%$ similarity. Within this cluster, a close similarity was indicated between isolates of: A, DTs 44, 49, 135 and 204c, with the distantly related DT 9; and B, between DTs 95 and 99 . Isolates of these phage types formed a subcluster within cluster I. The second subcluster C contained DTs 110,120 , and the more distantly related $104 \mathrm{a}$. The second cluster contained two subclusters: D, DTs 10, 20 and 146; and E, DTs 69, 103 and the distantly related 153 . The single isolate of DT 12 remained ungrouped in cluster II.

The ordination resulting from a principal coordinate analysis based upon the same data is shown in Fig. 3(b) (displaying $50.8 \%$ of the variance). The figure shows both the distances between the phage types in the space spanned by the principal axes as well as the nearest neighbours of the individual phage types as indicated by the MST. The result largely corresponds to the UPGMA clustering shown in Fig. 3(a), and the groups are encircled and lettered accordingly. Group (a) contained DTs 44, 49, 135 and 204c. DT 9 did not fall within any of the groups but its nearest neighbour was DT 44 of this group. Group B contained DTs 95 and 99. DTs 110 and 120 were closely connected in group C with DT 104a more distantly placed in the same group. Group D contained DTs 10, 20 and 146, but unlike in the cluster analysis, DT 12 was not connected to this group. Group E, like the subcluster E in Fig. 3(a), contained DTs 69, 103 and 153 . The nearest neighbour of DT 12 was DT 103 of this group.

The similarity matrices based upon the different typing techniques were compared. Table 4 shows the product moment correlation between the matrices indicating the relationships between results obtained by the different typing methods. The IS 200 similarity matrix correlated $81 \%$ with the matrix based on results of all three typing 
Table 4. Correlation coefficients between similarity matrices produced by three different typing methods and by pooled analysis of results from all three methods

\begin{tabular}{|lcccc|}
\hline Method & IS200 typing & Ribotyping & PFGE typing & All methods \\
\hline IS200 typing & 1.00 & 0.22 & 0.21 & 0.81 \\
Ribotyping & - & 1.00 & 0.47 & 0.69 \\
PFGE typing & - & - & 1.00 & 0.56 \\
All methods & - & - & - & 1.00 \\
\hline
\end{tabular}

methods, while the ribotype matrix and the PFGE matrix correlated $69 \%$ and $56 \%$ with this matrix.

\section{DISCUSSION}

Some phage types grouped together in the present investigation irrespective of the typing method used, suggesting the presence of different clones within the serotype $S$. typhimurium. The existence of such clones has previously been demonstrated by use of isoenzyme analysis (Beltran et al., 1988). The phage types investigated formed two groups with five subgroups according to the combined analysis of all typing methods. The groups were stably identified by UPGMA clustering, principal coordinate analysis and nearest neighbour analysis (Fig. 3a, b), which strongly suggests that the groupings observed represent real genetic relationships rather than incidental similarity of patterns. DT 9 was distantly connected to group A in the dendrogram (Fig. 3a) but was found spatially separated from this group in the ordination. It was, however, connected to the same group by the MST, suggesting that DT 9 is distantly related to DTs $44,49,135$ and 204c. DT 12 was connected to group $\mathrm{D}$ in the cluster analysis but to group $\mathrm{E}$ in the ordination, and the relationship between DT 12 and the other DTs has not been clearly solved by the present investigation.

All isolates joined each other at a similarity of approximately $67 \%$ in the UPGMA clustering. It is not clear how relatedness based on RFLP patterns relates to overall genetic similarity. Based on isoenzyme analysis of 23 enzymes, isolates of $S$. typhimurium show approximately $80 \%$ similarity (Beltran et al., 1988), but these authors did not report the phage type distribution, which makes comparison to the present investigation impossible. Compared with other serotypes of $S$. enterica, S. typhimurium seems to display a greater degree of diversity as indicated by both isoenzyme analysis (Beltran et al., 1988), and by the large span in IS200 copy number relative to copy number variation in other important serotypes (see Stanley et al., 1991, 1992; Olsen \& Skov 1994; Olsen et al., 1994).

The investigated isolates of DTs 110, 120 and 135 were very uniform, while a degree of genetic diversity was demonstrated for DT 49, possibly due to the broader strain collection used for the latter phage type. All isolates of these four phage types were found to form tight subclusters in the pooled analysis (data not shown), strongly suggesting that isolates of these phage types are clonally related. The remaining phage types were represented by selected isolates, and until further isolates have been analysed, the relationships demonstrated cannot be assumed to cover all isolates within a particular phage type.

It was assumed that the pooled dataset represented the most comprehensive information on similarity between the isolates of $S$. typhimurium, simply because more restriction sites were included. The individual ability of the three typing methods to indicate overall genetic relationships was evaluated against this dataset, and IS200 typing was found to be the best single method for such an analysis within S. typhimurium. In other important serotypes, such as Salmonella enteritidis and Salmonella dublin this may not be the case, as they in general contain only two or three copies of IS200 (Stanley et al., 1991; Chowdry et al., 1993; Olsen \& Skov, 1994; Olsen et al., 1994). Despite the fact that PFGE measures restriction sites in all areas of the chromosome, the results indicated that this method was the least suitable for analysis of relationships since a low degree of correlation was observed between the pooled dataset and PFGE $(r=0.56)$. This could be due to the choice of restriction enzymes and the fact that a relatively low number of fragments was analysed. Such large fragments, though of the same size, may contain different genetic information. Observations other than the low $r$ value pointed to PFGE as the least informative method: DT 204c grouped with the isolates of DTs 44, 49 and 135 by IS200 as well as by ribotyping. DT 204c probably belongs to the same genetic line as these phage types as it shows a close relationship with isolates of DT 49 as indicated by biotyping results (Barker \& Old, 1989 ), and phage type conversion from DT 49 to $204 \mathrm{c}$ has been reported (Threlfall et al., 1983). The close relationship between these two phage types was not indicated by PFGE. Furthermore, isolates of DT 49 with different PFGE patterns did not form a distinct subcluster, as had been demonstrated with the different IS200 types of the same phage type, but were widely spread in the dendrogram, suggesting that PFGE did not discriminate sufficiently between the isolates.

Results produced by IS200, ribotyping and PFGE resulted in slightly different grouping of some of the phage types. This observation is reflected in the lower $r_{\mathrm{cs}}$ value calculated for the combined analysis than for analysis based on just one technique, since the $r_{\mathrm{es}}$ value 
decreases when the dendrogram enforces a structure on the similarity data which is a compromise between different structures (Sneath \& Sokal, 1973). The observation indicates that the methods measure different parts of the chromosome, which have not evolved at the same rate, or that horizontal gene transfer and recombination in some areas of the chromosome has occurred between different clones of S. typhimurium. Such recombination events have been demonstrated between subspecies of $S$. enterica resulting in a degree of mosaic gene structure in flagellar antigen genes (Li et al., 1994). Recombination events have clearly also happened in the $r f b$ region encoding $\mathrm{O}$ antigens (Beltran et al., 1988), and it is believed to have been beneficial to the bacteria to exchange genes encoding surface structures. This is in contrast to genes encoding metabolic enzymes and housekeeping proteins, where recombination events seem to be rare (Nelson et al., 1991; Nelson \& Selander, 1992, 1994). The strains in the present investigation are of one serotype, and hence are identical in the genes where mosaic gene structure is common, and recombination events are unlikely to have caused the different grouping demonstrated by the three methods.

Typing by IS200 has commonly been used for evolutionary studies within different Salmonella serotypes. Isolates with the same copy number are assumed to share evolutionary traits, especially when the insertion elements are located within DNA fragments of the same size (Stanley et al., 1991, 1992). Stanley et al. (1993) therefore based their tentative phylogenetic tree of $S$. typhimurium mainly on the copy number of IS200. In our investigation, the overall similarity of IS200 profiles (Fig. 1a) correlated with the copy number of IS200 within cluster I of Fig. 1(a) (5-7 copies), while the copy number varied from seven to 20 in cluster II and from seven to 10 in cluster III. By comparing Fig. 1(a) with Fig. 3 it was also evident that grouping according to copy number of IS200 did not correlate strictly with the overall restriction pattern similarity between the isolates. The development of clonal lines of $S$. typhimurium is thus not directly reflected by the number of transposition events with IS200. However, IS200 typing was found to be the method that most closely reflected the overall similarity between the isolates. A possible explanation for these apparently conflicting observations is that some of the IS200 transposition events correlate with the evolution of clonal lines within S. typhimurium, but that additional and non-significant transposition events occur in some isolates, thereby creating IS200 patterns where the only difference is caused by the new copy. DT 49 was found to contain more than one IS200 profile, and unpublished results cited by Stanley et al. (1993) have shown that the copy numbers of IS 200 differ within DT 204c. As our results also show a different copy number for IS200 compared with the results of Stanley et al. (1993) in the case of DTs 49 and 12, the IS200 copy number may vary so much within certain phage types that it is unsuitable as a main marker for genetic relationships between strains. Within those phage types where several IS200 patterns are present, e.g. DT 49, hybridization with IS200 provides a means for subtyping. This approach has already been applied for comparison of isolates of S. typhimurium and $S$. enteritidis of avian origin (Millemann et al., 1995).

Ribotyping was originally suggested as a method for taxonomic studies (Grimont \& Grimont, 1986), but is now more commonly used for typing purposes. Comparison of ribotype patterns between different analyses are impossible due to differences in probes used. For example, Stanley et al. (1993) based conclusions on phylogeny of $S$. typhimurium phage types from ribotyping using just one enzyme and a probe complementary to a $550 \mathrm{bp}$ internal fragment of $16 \mathrm{~S}$ rRNA, and found that all clinical isolates of S. typhimurium were of the same ribotype. More information is obtained when more than one enzyme is used for the characterization, and when the probe used for hybridization covers both the $16 \mathrm{~S}$ and $23 \mathrm{~S}$ rRNA subunits, as was the case in the present investigation. There is clearly a need for standardization within this area to maximize the ability to compare results from different laboratories.

\section{ACKNOWLEDGEMENTS}

This study was supported by the Danish Agricultural and Veterinary Research Council (grant no. 20-3510-1) and by the Danish Food Research Centre. We are grateful to Gitte Christensen, Anita Forslund, Tony Bønnelycke and Jan Pedersen for technical assistance. Derek Brown is thanked for critical reading of this manuscript.

\section{REFERENCES}

Anderson, E. S., Ward, L. R. \& de Saxe, M. J. (1977). Bacteriophage-typing designations of Salmonella typhimurium. J Hyg 78, 297-300.

Baggesen, D. L. \& Wegener, H.C. (1994). Phage types of Salmonella enterica spp. enterica serovar typhimurium isolated from production animals and humans in Denmark. Acta Vet Scand 35, 349-354.

Barker, R. M. \& Old, D. C. (1989). The usefulness of biotyping in studying the epidemiology and phylogeny of salmonellae. J Med Microbiol 29, 81-88.

Beltran, P., Musser, J. M., Helmuth, R. \& 8 other authors (1988). Towards a population genetic analysis of Salmonella: genetic diversity and relationships among isolates of serotypes $S$. choleraesuis, S. derby, S. dublin, S. enteritidis, S. heidelberg, S. infantis, S. newport and S. typhimurium. Proc Natl Acad Sci USA 85, 7753-7757.

Callow, B. R. (1959). A new phage-typing scheme for Salmonella typhimurium. J Hyg 57, 346-359.

Cameron, D. N., Khambaty, F. M., Wachsmuth, I. K., Tauxe, R. V. \& Barret, T. J. (1994). Molecular characterization of Vibrio cholerae $\mathrm{O} 1$ isolates by pulsed-field gel electrophoresis. J Clin Microbiol 32, 1685-1690.

Chowdry, N., Threlfall, E. J., Rowe, B. \& Stanley, E. J. (1993). Genotype analysis of faecal and blood isolates of Salmonella dublin from humans in England and Wales. Epidemiol Infect 110, 217-225.

Christensen, J. P., Olsen, J. E. \& Bisgaard, M. (1993). Ribotypes of Salmonella enterica serovar Gallinarum biovars gallinarum and pullorum. Avian Pathol 22, 725-738. 
Gibert, I., Barbé, J. \& Casadesús, J. (1990). Distribution of insertion sequence IS200 in Salmonella and Shigella. J Gen Microbiol 136, 2555-2560.

Gower, J. C. (1966). Some distance properties of latent root and vector methods used in multivariate analysis. Biometrica 53, 325-338.

Grimont, F. \& Grimont, P. A. D. (1986). Ribosomal ribonucleic acid gene restriction patterns as potential taxonomic tools. Ann Inst Pasteur Microbiol 137B, 165-175.

Li, J., Nelson, K., McWhorter, A. C., Whittam, T. S. \& Selander, R. K. (1994). Recombinational basis of serovar diversity in Salmonella enterica. Proc Natl Acad Sci USA 91, 2552-2556.

Maniatis, T., Fritsch, E. F. \& Sambrook, J. (1982). Molecular Cloning: a Laboratory Manual. Cold Spring Harbor, NY: Cold Spring Harbor Laboratory.

Millemann, Y., Lesage, M.-C., Chaslus-Dancla, E. \& Lafont, J.-P. (1995). Value of plasmid profiling, ribotyping, and detection of IS200 for tracing avian isolates of Salmonella typhimurium and S. enteritidis. J Clin Microbiol 187, 157-167.

Nelson, K. \& Selander, R. K. (1992). Evolutionary genetics of the proline permease gene $(p u t P)$ and the control region of the proline utilization operon in populations of Salmonella and Escherichia coli. J Bacteriol 174, 6886-6895.

Nelson, K. \& Selander, R. K. (1994). Intergeneric transfer and recombination of the 6-phosphogluconate dehydrogenase gene (gnd) in enteric bacteria. Proc Natl Acad Sci USA 91, 10227-10231.

Nelson, K., Whittam, T. S. \& Selander, R. K. (1991). Nucleotide polymorphisms and evolution in the glyceraldehyde-3-phosphate dehydrogenase gene $(\mathrm{g} a \mathrm{p} A)$ in natural populations of Salmonella and Escherichia coli. Proc Natl Acad Sci USA 88, 6667-6671.

Olsen, J. E. (1990). An improved method for rapid isolation of plasmid DNA from wild-type Gram-negative bacteria for restriction profile analysis. Lett Appl Microbiol 10, 209-212.

Olsen, J. E. \& Skov, M. (1994). Genomic lineage of Salmonella enterica serovar Dublin. Vet Microbiol 40, 271-282.
Olsen, J. E., Skov, M. N., Threlfall, E. J. \& Brown, D. J. (1994). Clonal lines of Salmonella enterica serotype Enteritidis documented by IS200, ribo, pulsed field gel electrophoresis and RFLP typing. J Med Microbiol 40, 15-22.

Rohlf, F. J. (1993). NTSYS-pc. Numerical Taxonomy and Multivariate Analysis System. New York: Applied Biostatistics.

Sneath, P. H. A. \& Sokal, R. R. (1973). Numerical Taxonomy. San Francisco: Freeman.

Stanley, J., Jones, C. \& Threlfall, E. J. (1991). Evolutionary lines of $S$. enteritidis phage types are identified by insertion sequence IS200 distribution. FEMS Microbiol Lett 82, 83-90.

Stanley, J., Chowdry, N., Powell, N. \& Threlfall, E. J. (1992). Chromosomal genotypes (evolutionary lines) of Salmonella berta. FEMS Microbiol Lett 95, 247-252.

Stanley, J., Baquar, N. \& Threlfall, E. J. (1993). Genotypes and phylogenetic relationships of Salmonella typhimurium are defined by molecular fingerprinting of IS200 and $16 \mathrm{~S} \mathrm{rrn}$ loci. J Gen Microbiol 139, 1133-1140.

Threlfall, E. J., Ward, L. R. \& Rowe, B. (1983). The use of phage typing and plasmid characterization in studying the epidemiology of multi resistant Salmonella typhimurium. In Antibiotics: Assessment of Antimicrobial Activity and Resistance, pp. 285-297. Edited by A. Russel \& L. Quesnel. London: Academic Press.

Threlfall, E. J., Brown, D. J., Rowe, B. \& Ward, L. R. (1990a). Occurrence of S. typhimurium DT204C in poultry in England and Wales. Vet Rec 127, 234.

Threlfall, E. J., Frost, J. A., Ward, L. R. \& Rowe, B. (1990b). Plasmid profile typing can be used to subdivide phage type 49 of Salmonella typhimurium in outbreak investigations. Epidemiol Infect 104, 243-251.

Received 14 June 1996; revised 29 October 1996; accepted 26 November 1996. 